\title{
Note on the Geometry of the Triangle.
}

\author{
By John Miller.
}

\section{(Read 12th February 1909).}

1. The following note examines, more closely than the text books usually do, some results of reciprocation, and has special reference to properties of a certain infinite set of triangles and connected conics.

2. Reciprocate a triangle $A^{\prime} B^{\prime} C^{\prime}$ with respect to its circumcircle of centre S. A triangle $\mathrm{ABC}$ is obtained circumscribed about the same circle. All other circles, as is well known, reciprocate into conics with $S$ as one focus; thus the four inscribed and escribed circles of $\mathrm{A}^{\prime} \mathrm{B}^{\prime} \mathrm{C}^{\prime}$ become four circumconics of $\mathrm{ABC}$ with $\mathrm{S}$ as one focus. Pairs of perpendicular lines become pairs of points subtending a right angle at $\mathrm{S}$. Hence the orthocentre of $\mathrm{A}^{\prime} \mathrm{B}^{\prime} \mathrm{C}^{\prime}$ becomes a transversal $\mathrm{XYZ}$ of $\mathrm{ABC}$ where $\mathrm{SX}$ is perpendicular to $\mathrm{SA}, \mathrm{SY}$ to $\mathrm{SB}$ and $\mathrm{SZ}$ to $\mathrm{SC} ; \mathbf{S}\{\mathbf{A X}, \mathbf{B Y}, \mathbf{C Z}\}$ is a pencil in involution. If a line in the original figure be divided in a given ratio, by including the point at infinity on the line, we obtain, on reciprocating, a pencil with a given anharmonic ratio, one of the rays passing through $S$. Thus the centroid of any triangle becomes the trilinear polar of $S$ with respect to the reciprocal triangle. For $\mathrm{ABC}$ this trilinear polar $\mathrm{X}^{\prime} \mathrm{Y}^{\prime} \mathrm{Z}^{\prime}$ is the line joining the intersections with the opposite sides of the external bisectors of the angles of $A B C$. The centre of the nine point circle of $A^{\prime} B^{\prime} C^{\prime}$ becomes the directrix of a nine line ellipse, the corresponding focus being $\mathbf{S}$. This line and the lines $\mathrm{XYZ}, \mathrm{X}^{\prime} \mathrm{Y}^{\prime} \mathrm{Z}^{\prime}$ are parallel, and if a perpendicular through $\mathrm{S}$ cut them in $\mathrm{S}^{\prime}, \mathrm{H}, \mathrm{G}$ respectively, $\mathrm{SH}=\mathbf{H} \mathbf{S}^{\prime}$ and $2 \mathrm{SH}=\mathrm{HG}$.

3. Let $S$ be the centre of a circle inscribed in $A B C$ and touching the sides in $\mathbf{A}^{\prime}, \mathbf{B}^{\prime}, \mathbf{C}^{\prime}$. Let $\mathrm{AS}, \mathrm{BS}, \mathrm{CS}$ cut the opposite sides in $D, E, F$. Consider the involution $\left\{B C, A^{\prime} D, \ldots\right\}$. This is the 
reciprocal of the involution of the isogonal conjugates through $\mathrm{A}^{\prime}$ in the triangle $A^{\prime} B^{\prime} C^{\prime}$. The tangents from points on $\mathrm{SA}$ cut $\mathrm{BC}$ in pairs of corresponding points, so that the double points $\mathbf{L}, \mathbf{M}$ are the intersections with $\mathrm{BC}$ of the tangents at the ends of the diameter on SA; let $L$ be internal and $M$ external. If $N, P$ and $\mathrm{Q}, \mathrm{R}$ be the double points of the similar involutions on $\mathrm{CA}$ and $\mathrm{AB}$, we see that the double points lie in sets of three on four lines RNL, NQM, LQP, PRM. These lines are the reciprocals of the in- and ex-centres of $\mathrm{A}^{\prime} \mathrm{B}^{\prime} \mathrm{C}^{\prime}$ and are the directrices corresponding to the focus $S$ of the four circumscribing conics of $\mathrm{ABC}$ mentioned above. $A B C$ is the diagonal triangle of the quadrilateral formed by the four lines. If a transversal cut $\mathrm{ABC}$, the three corresponding points in the three involutions lie on a corresponding line, and the corresponding lines of a pencil envelop an inseribed conic. The correspondent of the line at infinity is the line $\mathrm{XYZ}$ above joining the centres $X, Y, Z$ of the three involutions. If two corresponding lines $o, o^{\prime}$ cut $\mathrm{SX}, \mathrm{SY}, \mathrm{SZ}$ in $\mathrm{O}_{x} \mathrm{O}_{z}^{\prime}, \mathrm{O}_{n}, \mathrm{O}_{y}^{\prime}, \mathrm{O}_{z} \mathrm{O}_{z}^{\prime}$, then $\mathrm{AO}_{x}, \mathrm{AO}_{x}^{\prime}, \mathrm{BO}_{y}, \mathrm{BO}_{y}^{\prime}, \mathrm{CO}_{z}, \mathrm{CO}_{z}^{\prime}$ touch a conic with $\mathrm{S}$ as a focus, the corresponding directrix being the harmonic conjugate of SK to $o$ and $o^{\prime}$ where $\mathrm{K}$ is the intersection of $o$ and $o^{\prime}$.

The trilinear polar of the Brianchon point of $\mathrm{ABC}$ corresponds to $\mathrm{X}^{\prime} \mathrm{Y}^{\prime} \mathrm{Z}^{\prime}$.

The nine line ellipse is one of this set of conics, the corresponding lines being XYZ and the line at infinity.

The pairs of double points $I_{,} M ; N, P ; Q, R$ subtend right angles at $S$ since they are the reciprocals of the lines of centres of the inscribed and escribed circles of $A^{\prime} B^{\prime} C^{\prime}$. $S$ is therefore one of the common points of the director circles of the conics inscribed in the quadrilateral RNQP ; the other common point is $S^{\prime}$, the foot of the perpendicular from $S$ on the directrix of the nine line ellipse corresponding to $\mathrm{S}$ since $\mathrm{XYZ}$ is the line of centres of these conics. The circumcircle of $\mathrm{ABC}$ cuts all these director circles orthogonally,

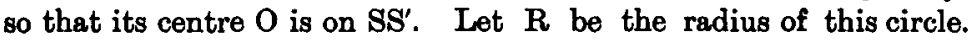
Then $\mathrm{OS}^{\circ} \mathrm{OS}^{\prime}=\mathrm{R}^{2}$. If $r$ is the radius of the inscribed circle of $A B C$, the square of the distance of $S$ from the orthocentre of of $\mathrm{A}^{\prime} \mathrm{B}^{\prime} \mathrm{C}^{\prime}$, which lies on $\mathrm{SS}^{\prime}$, is

$r^{2}\left(1-8 \cos A^{\prime} \cos B^{\prime} \cos C^{\prime}\right)=r^{2}\left(1-8 \sin \frac{A}{2} \sin \frac{B}{2} \sin \frac{C}{2}\right)=\frac{r^{2}}{R^{2}}\left(R^{3}-2 R r\right)$. 


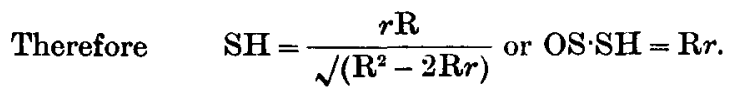

Let $r_{0}$ be the inradius of $\mathrm{A}^{\prime} \mathrm{B}^{\prime} \mathrm{C}^{\prime}$.

The distance of $\mathbf{S}$ from the directrix of the circumscribed conic (i) of $\mathrm{ABC}$, which is the reciprocal of the inscribed circle of $\mathrm{A}^{\prime} \mathrm{B}^{\prime} \mathrm{C}^{\prime}$ is $\frac{r^{2}}{\sqrt{\left(r^{2}-2 r r_{0}\right)}}$.

The distance of the centre of (i) from the directrix is

$$
\frac{r^{2} r_{0}^{2}}{\left(r_{0}^{2}-r^{2}+2 r r_{0}\right) \sqrt{\left(r^{2}-2 r r_{0}\right)}}
$$

and the eccentricity is $\frac{\sqrt{ }\left(r^{2}-2 r r_{0}\right)}{r_{0}}$.

The semi-transverse axis is $\frac{r^{2} r_{0}}{r_{0}^{2}-r^{2}+2 r r_{0}}$ und the semi-conjugate axis is $\frac{r^{2}}{\sqrt{ }\left(r_{0}^{2}-r^{2}+2 r r_{0}\right)}$.

Corresponding results for the other three conics (ii), (iii), (iv) are obtained by writing $-r_{1},-r_{2},-r_{3}$ for $r_{0}$ where $r_{1}, r_{2}, r_{3}$ are the radii of the escribed circles of $\mathrm{A}^{\prime} \mathrm{B}^{\prime} \mathrm{C}^{\prime}$.

The sum of the squares of the reciprocals of the distances of $\mathrm{S}$ from the four corresponding directrices is $\frac{12}{r^{2}}$. The sum of the reciprocals of the transverse axes is $\frac{8}{r}$. The latus rectum of (i) is $\frac{2 r^{2}}{r_{0}}$ and is equal to the sum of the latera recta of (ii), (iii), (iv). The sum of the reciprocals of the last three lines diminished by the reciprocal of the latus rectum of (i) is $\frac{2}{r}$, which is equal to half the sum of the reciprocals of the semi-transverse axes.

These sums are then the same for all the triangles circumscribing the same circle $\mathrm{S}$.

4. Consider the nine line ellipse whose focus is $S$ and whose corresponding directrix is the line through $\mathbf{S}^{\prime}$ parallel to XYZ. It is touched by $\mathrm{AX}, \mathrm{AX}^{\prime}, \mathrm{BY}, \mathrm{BY}^{\prime}, \mathrm{CZ}, \mathrm{CZ}^{\prime}$. The harmonic 
conjugate of $\mathrm{XS}$ with respect to $\mathrm{BC}$ and $\mathrm{XYZ}$ is also a tangent; similar tangents are found through $\mathrm{Y}$ and $Z$. The ellipse also touches the four circumscribing conics.

The distance $\mathrm{SS}^{\prime}$ is $\frac{2 \mathrm{R} r}{\sqrt{\left(\mathrm{R}^{2}-2 \mathrm{R} r\right)}}$.

The distance squared of $\mathrm{S}$ from the nine point centre of $\mathrm{A}^{\prime} \mathrm{B}^{\prime} \mathrm{C}^{\prime}$ is $\frac{r^{2}}{4}\left(1-8 \cos \mathrm{A}^{\prime} \cos \mathrm{B}^{\prime} \cos \mathrm{C}^{\prime}\right)=\frac{r^{2}}{4}\left(1-8 \sin \frac{\mathrm{A}}{2} \sin \frac{\mathrm{B}}{2} \sin \frac{\mathrm{C}}{2}\right)=\frac{r^{2}}{4}\left(1-\frac{2 r}{\mathrm{R}}\right)$.

Therefore the distance of the polar of $\mathrm{S}$ with respect to the nine point circle of $\mathrm{A}^{\prime} \mathrm{B}^{\prime} \mathrm{C}^{\prime}$ from $\mathrm{S}$ is $\overline{\left.\sqrt{\left(\mathrm{R}^{2}\right.}-2 \mathrm{R} r\right)}$.

Hence the distance of the centre of the nine line ellipse from $\mathrm{S}$ is $\sqrt{ }\left(\mathbf{R}^{2}-2 R r\right)$ which is equal to SO.

The centre of the circumcircle and the nine line ellipse of $\mathrm{ABC}$ then coincide. The distance of this centre from $\mathrm{S}^{\prime}$ being $\frac{\mathrm{R}^{2}}{\sqrt{ }\left(\mathrm{R}^{2}-2 \mathrm{R} r\right)}$ the eccentricity is $\frac{\sqrt{ }\left(\mathrm{R}^{2}-2 \mathrm{R} r\right)}{\mathrm{R}}$.

The semi-major axis is $\mathrm{R}$ and the semi-minor axis is $\sqrt{ }(2 \mathrm{R} r)$; the semi-latus rectum is $2 r$. The auxiliary circle of the ellipse is therefore the circumcircle of $\mathrm{ABC}$.

Consider the infinite set $(a)$ of triangles inscribed in the circle with centre $O$ and radius $R$ and circumscribed about the circle with centre $S$ and radius $r$. [To get the circles take an ellipse with its auxiliary circle and with a focus as centre and half the semi-latus rectum as radius, describe a second circle.] These have $O, S, S^{\prime}, H$ and the lines $\mathrm{XYZ}, \mathrm{X}^{\prime} \mathrm{Y}^{\prime} \mathrm{Z}^{\prime}$ in common. Thus the perpendiculars from $S$ to the lines joining $S$ to the vertices cut the opposite sides in points lying on the same line XYZ, and the external bisectors cut the opposite sides in points all lying on another line $X^{\prime} Y^{\prime} Z^{\prime}$. The set (a) have the same nine line ellipse which is evidently the envelope of their external bisectors. It is also the envelope of the lines $\mathrm{AX}$, etc.

Since the distance between $\mathbf{S}$ and the centres of the nine point circles of the set $(a)$ is $\frac{1}{2} R-r$, these centres lie on a circle with centre $\mathbf{S}$, and the envelope of the circles is two circles with centre $\mathbf{S}$. The loci of the centroids and orthocentres of the set (a) are two circles with centres on OS and radii $\frac{1}{3}(R-2 r),(R-2 r)$. 
On reciprocating this set $(\alpha)$ with respect to $S$ we see that the infinite set $(\beta)$ of triangles inscribed in the same circle of centre $\mathbf{S}$ and having the same orthocentre $K$ have their sides enveloping a conic with $\mathrm{S}$ as a focus, the corresponding directrix being perpendicular to SK.

5. Let $o$, any tangent to the inscribed circle of $\mathrm{ABC}$, cut $\mathrm{SX}$, $\mathrm{SY}, \mathrm{SZ}$ in $\mathrm{O}_{x}, \mathrm{O}_{y}, \mathrm{O}_{z} ; \mathrm{AO}_{x}, \mathrm{BO}_{y}, \mathrm{CO}_{z}$, meet in a (Wallace) point $\mathrm{W}$.

The Wallace points of two parallel tangents lie on a tangent to the nine line ellipse and subtend a right angle at $S$.

The locus of $W$ for all tangents is a cubic of class four (the reciprocal of the three cusped hypocycloid) with three real points of inflexion on the directrix of the nine line ellipse through $\mathrm{S}^{\prime}$. It passes through $\mathrm{A}, \mathrm{B}, \mathrm{C}, \mathrm{X}, \mathrm{Y}, \mathrm{Z}$, and has triple contact with the ellipse. 